\title{
Vigilância Sanitária e segurança da atenção materna e neonatal: proposta de modelo lógico
}

\author{
Health Surveillance and safety in maternal and neonatal care: \\ proposal of logic model
}

Ana Maria Bourguignon ${ }^{1, *}$ (iD)

Zulmira Hartz" iD

Dirceia Moreira' iD

Universidade Estadual de Ponta Grossa (UEPG), Ponta Grossa, PR, Brasil

" Instituto de Higiene e Medicina Tropical, Universidade Nova de Lisboa, Lisboa, Portugal

\footnotetext{
* E-mail: anamariabourg@gmail.com
}

Recebido: 15 jun 2020

Aprovado: 20 out 2020

\section{RESUMO}

Introdução: A promoção da segurança do paciente em serviços de atenção materna e neonatal constitui área prioritária para a política de saúde no Brasil, porque contribui para a redução da morbimortalidade de mulheres e recém-nascidos. Objetivo: Elaborar um modelo lógico com base no manual Serviços de Atenção Materna e Neonatal: Segurança e Qualidade, no qual a Agência Nacional de Vigilância Sanitária compila recomendações específicas para os serviços cujo público-alvo são mulheres em estado gravídico e puerperal, bem como seus filhos e filhas. Método: Trata-se de um estudo qualitativo, descritivo, que utilizou como procedimento metodológico a análise documental. A avaliação normativa procurou responder a um roteiro com doze questões elaboradas por Bezerra et al. para modelização de programas. Resultados: Foi evidenciada a necessidade de integração entre Vigilância Sanitária e os serviços de assistência obstétrica e neonatal, para que os efeitos almejados sejam alcançados. Conclusões: 0 modelo lógico proposto pode ser uma ferramenta útil, tanto para a organização e permanente monitoramento dos serviços, como para os profissionais que atuam na Vigilância Sanitária. Além de potencializar o reconhecimento da contribuição da vigilância sanitária para a promoção da saúde materno-infantil.

PALAVRAS-CHAVE: Avaliação em Saúde; Vigilância Sanitária de Serviços de Saúde; Segurança do Paciente; Saúde Materno-Infantil; Brasil

\begin{abstract}
Introduction: The promotion of patient safety in maternal and neonatal care services is a priority area for health policy in Brazil, because it contributes to reducing the morbidity and mortality of women and newborns. Objective: To elaborate a logical model based on the handbook Maternal and Neonatal Care Services: Safety and Quality, in which the National Health Surveillance Agency compiles specific recommendations for services whose target audience is pregnant and puerperal women, as well as their sons and daughters. Method: This is a qualitative, descriptive study, which used documentary analysis as a methodological procedure. The normative assessment sought to answer a script with twelve questions prepared by Bezerra et al. for program modeling. Results: It was evidenced that the integration between Health Surveillance and obstetric and neonatal care services is necessary, so that the desired effects can be achieved. Conclusions: The proposed logical model can be a useful tool, both for the organization and permanent monitoring of services, and for professionals working in Health Surveillance. Additionally, it may enhance the recognition of the health surveillance contribution to the promotion of maternal and infant health.
\end{abstract}

KEYWORDS: Health Evaluation; Health Surveillance of Health Services; Patient Safety; Maternal and Child Health; Brazil 


\section{INTRODUÇÃO}

A promoção da saúde da mulher e do recém-nascido durante o ciclo gravídico-puerperal demanda a atuação de diferentes níveis de assistência e de órgãos distintos das estruturas governamentais. A atenção primária mantém o contato direto com as usuárias e suas famílias, responde pela vigilância da gravidez e do puerpério, e promove o planejamento familiar, puericultura e a educação em saúde. A atenção terciária presta os cuidados obstétricos e neonatais necessários em caso de intercorrência, bem como a assistência ao parto e os cuidados nas primeiras horas após o nascimento. Transversal a ambos os níveis de atenção, o sistema de vigilância sanitária tem por finalidade promover a proteção da saúde da população, mediante o controle sanitário da produção e circulação de bens e da prestação de serviços, incluindo aspectos relativos a ambientes, processos, insumos e tecnologias ${ }^{1}$. Nesse amplo raio de atuação, competências específicas distinguem a atuação da vigilância sanitária no sentido de promover a saúde materna e neonatal.

A relação entre vigilância sanitária e a saúde materna, entretanto, nem sempre é evidente. 0 estudo realizado por Maia et al. ${ }^{2}$ entrevistou profissionais vinculados às secretarias de saúde de municípios capitais de estado, um de cada região do Brasil. No total, participaram 15 profissionais, dos quais cinco coordenadores de saúde da mulher, cinco responsáveis e cinco técnicos pela Vigilância Sanitária (Visa) de serviços de saúde. O primeiro grupo de profissionais considerou a integração entre as áreas importante, porém difícil de ser executada. Na perspectiva dos trabaIhadores da Visa, a atuação em conjunto com a área de saúde da mulher acontecia apenas a partir de demandas pontuais.

Os pesquisadores constataram a fragmentação entre as áreas das secretarias e isolamento da Visa, com a consequente dissociação entre as ações de assistência e de prevenção, "[...] seja em razão do entendimento de que uma não depende da outra, seja porque nunca se pensou nessa possibilidade de um trabatho conjunto ou porque não houve apresentação de uma área a outra" 2 . O resultado é corroborado pela pesquisa qualitativa de Fernandes e Vilela ${ }^{3}$. A análise das entrevistas com 11 gestores de Visa e de saúde da mulher, vinculados a quatro municípios localizados no estado de São Paulo, concluiu que "[...] as práticas de Visa ainda se encontram isoladas das demais práticas de saúde e que, muitas vezes, são identificadas apenas como práticas fiscalizatórias" ${ }^{3}$.

No que se refere à atenção neonatal, a pesquisa de Tomazoni et al. ${ }^{4}$ avaliou 12 dimensões da segurança do paciente na perspectiva de 181 profissionais de equipes de enfermagem e medicina de unidades de terapia intensiva neonatal. As dimensões com respostas predominantemente positivas foram: "expectativas e ações do supervisor/chefia para a promoção da segurança do paciente" (61\%); "aprendizado organizacional - melhoria contínua" (59\%); "trabalho em equipe na unidade" (57\%); “abertura para comunicação” (55\%). Outras dimensões com maiores proporções de respostas negativas: "resposta não punitiva ao erro" (58\%), a maioria dos respondentes afirmou que, na hipótese de um erro notificado, o funcionário fica exposto e não o problema; "apoio da gestão hospitalar para segurança do paciente" (51\%), a maior parte discordou que suas chefias estimulam ações para promoção de segurança; "quadro de funcionários", considerado por parte significativa dos profissionais como insuficiente para a demanda (43\%); "trabalho em equipe" entre as unidades hospitalares (42\%), muitos entrevistados avaliaram não haver interação entre as diferentes unidades do hospital. Os resultados da pesquisa indicam que a cultura organizacional interfere na promoção da segurança do paciente nos serviços de atenção neonatal e reforça a necessidade de ampliar os estudos sobre a temática.

Embora a vigilância sanitária tenha um caráter estratégico para a proteção da saúde da população - atuando na eliminação, diminuição e prevenção dos riscos à saúde -, não existe uma política pública específica para a área no Brasil ${ }^{5,6}$. Depreende-se que a amplitude do espectro de atuação da Visa e a ausência de uma política específica para o setor contribuem para as dificuldades de integração da área com outros segmentos da gestão em saúde, em especial com a saúde materna e perinatal. Ademais, a falta de clareza quanto às metas a serem alcançadas e de indicadores de saúde relacionados à atuação da Visa inviabilizava a concretização da etapa de avaliação pertinente ao ciclo da política pública.

A partir dessa constatação e com o intuito de elucidar a teoria e a lógica subjacentes ao Sistema Nacional de Vigilância Sanitária (SNVS), Felisberto et al. ${ }^{6}$ executaram um projeto de modelagem avaliativa com enfoque na construção de indicadores das ações de vigilância sanitária. Primeiramente, construiu-se o modelo teórico da intervenção, entendida como "um sistema organizado de ação, constituído a partir da priorização daquelas atividades mais representativas em execução pelo Sistema [...]"6. O modelo teórico procura identificar como uma intervenção funciona e os fatores de contexto que podem influir no seu funcionamento. Assim, definiu-se que o SNVS é a intervenção responsável pelo conjunto das "Ações de Vigilância Sanitária", integrado por cinco componentes: gestão; regulação; controle sanitário; monitoramento do risco sanitário; informação, comunicação e educação para a saúde. 0 modelo teórico também aponta os impactos, isto é, os resultados a nível populacional de longo prazo, esperados com atividades desenvolvidas pela Visa: redução da morbidade e da mortalidade; proteção e promoção da saúde; reconhecimento social da Visa.

O modelo lógico descrito por Felisberto et al. ${ }^{6}$ complementa a modelagem teórica sintetizada acima, na medida em que estabelece as conexões entre os componentes, subcomponentes, atividades, efeitos intermediários, impactos e indicadores de saúde vinculados à atuação da Visa. Recomenda-se visualizar o desenho esquemático do SNVS, resultante da modelagem avaliativa empreendida, no documento final intitulado "Avaliação das Ações de Vigilância Sanitária: uma proposta teórico-metodológica" 5 . 0 referido modelo teórico-lógico ${ }^{5}$ possibilita um novo patamar para a discussão e avaliação das atribuições pertinentes à Visa. 
Com base nesse pressuposto e na premissa que a atuação da Visa contribui para a melhoria dos indicadores de saúde relativos à qualidade e à segurança dos serviços de assistência obstétrica e neonatal, o presente estudo objetivou apresentar um modelo lógico do manual intitulado "Serviços de Atenção Materna e Neonatal: Segurança e Qualidade"7. O manual tem por escopo orientar medidas para aumentar a segurança do paciente e a qualidade dos serviços de saúde, especificamente, no âmbito da assistência materna e neonatal.

\section{MÉTODO}

O presente estudo dedicou-se a analisar as recomendações para a implementação de um Programa para a Promoção da Qualidade e Segurança na Atenção Materna e Neonatal (PPQSAMN). As recomendações estão disponíveis no manual da Agência Nacional de Vigilância Sanitária (Anvisa), intitulado Serviços de Atenção Materna e Neonatal: Segurança e Qualidade, que foi publicado em 2014. Trata-se, portanto, de um estudo qualitativo que utilizou a pesquisa documental como procedimento metodológico.

Um programa se constitui por um sistema organizado de ações direcionadas a intervir, num período específico de tempo, em uma situação problemática circunscrita a determinado contexto ${ }^{8}$. Haja vista que nem todos os programas trazem o modelo teórico-lógico explicitamente, como é o caso do PPQSAMN ${ }^{7}$, a modelização é considerada etapa essencial pela literatura para a realização de estudos avaliativos ${ }^{8,9,10,11,12}$.

As questões formuladas por Bezerra et al. ${ }^{8}$ para a modelização de programas serviu de roteiro base para analisarmos os parâmetros preconizados para a instituição de um PPQSAMN nos serviços de atenção materna ${ }^{7}$. $O$ roteiro contemplou os seguintes aspectos: 1) situação-problema que demandou o programa; 2) descrição do programa instituído para enfrentar o problema; 3) objetivos, metas e público-alvo do programa; 4) componentes; 5) estrutura necessária; 6) ações previstas; 7) produtos/resultados esperados; 8) fatores internos e externos que influenciam o alcance dos resultados.

A aplicação do roteiro foi apresentada em forma de diagrama para otimizar a comunicação com possíveis atores envolvidos com o planejamento, a execução, o monitoramento e a avaliação dos serviços de atenção materna no Brasili1. Ademais, dialoga com o modelo teórico-lógico das ações de vigilância sanitária presente nas recomendações da Anvisa ${ }^{5}$.

A análise do manual da Anvisa para a implementação do PPQSAMN foi complementada pela consulta a outras fontes normativas de apoio: a) Portaria Gabinete do Ministro/Ministério da Saúde (MS) $\mathrm{n}^{\circ} 529$, de $1^{\circ}$ de abril de $2013^{13}$, que instituiu o Programa Nacional de Segurança do Paciente (PNSP); b) Resolução da Diretoria Colegiada (RDC) Anvisa $n^{\circ} 36$, de 25 de julho de $2013^{14}$, referente às ações para a segurança do paciente em serviços de saúde; c) RDC Anvisa $n^{\circ} 36$, de 3 de junho de $2008^{15}$, que dispôs sobre o regulamento técnico para funcionamento dos serviços de atenção obstétrica e neonatal.
A consulta a essas normas ocorreu na medida em que fundamentam as recomendações para a segurança materna e neonatal. A Portaria GM/MS n ${ }^{\circ} 529 / 2013^{13}$ é citada logo na introdução do manual analisado para indicar a responsabilidade da Anvisa em coordenar a implementação do PNSP. Das ações dispostas na RDC Anvisa $n^{\circ} 36 / 2013^{14}$, destaca-se a recomendação para instituição e funcionamento dos Núcleos de Segurança do Paciente nos serviços de saúde. Ademais, os recursos estruturais necessários para o funcionamento dos serviços de atenção obstétrica e neonatal são apresentados no manual em conformidade aos padrões de qualidade, de segurança e de humanização previstos na RDC Anvisa $n^{\circ} 36 / 2008^{15}$. Portanto, as normas consultadas subsidiam o PPQSAMN, cujo modelo lógico é apresentado e discutido a seguir.

\section{RESULTADOS E DISCUSSÃO}

O SNVS é coordenado pela Anvisa, agência reguladora criada pela Lei $\mathrm{n}^{\circ}$ 9.782, de 26 de janeiro de 1999'1, sob regime de autarquia especial caracterizada pela autonomia financeira, independência administrativa e estabilidade de seus dirigentes, vinculada ao MS. Dentre as atribuições previstas em lei, à Anvisa compete "estabelecer normas, propor, acompanhar e executar as políticas, as diretrizes e as ações de vigilância sanitária", com abrangência em todo o território nacional. Desse modo, a agência responde pelas políticas nacionais de vigilância sanitária que impactam na promoção da saúde de diversos grupos populacionais, especificamente, para o interesse deste artigo, a saúde da mulher durante o ciclo gravídico-puerperal e do neonato.

A Anvisa estabelece parâmetros, dispostos em normas e guias, para o funcionamento de estabelecimentos e serviços que prestam assistência obstétrica e neonatal. A publicação intitulada Serviços de Atenção Materna e Neonatal: Segurança e Qualidade $^{7}$, realizada pela agência em parceira com a Organização Pan-Americana de Saúde (OPAS), apresenta um conjunto de recomendações e elenca as etapas necessárias para a implementação de um PPQSAMN. O programa é aplicável "a qualquer local onde se oferece assistência materna e neonatal", independentemente do regime jurídico, incluindo serviços primários de atenção - a exemplo das unidades básicas de saúde, ambulatórios, centros de parto normal, serviços hospitalares e espaços assistenciais como as Casas das Gestantes, Bebês e Puérperas. Considerando as especificidades e o contexto de cada serviço, adaptações ao programa proposto pela Anvisa podem ser ponderadas pelas respectivas equipes locais ${ }^{7}$.

0 contexto de elaboração do manual remete à preocupação em prevenir erros no processo assistencial que possam acarretar danos aos usuários de serviços de saúde. A Aliança Mundial para Segurança do Paciente foi lançada em 2004 pela Organização Mundial da Saúde (OMS) como forma de obter o comprometimento dos Estados signatários, dentre eles o Brasil, com a instituição de medidas para melhorar a qualidade dos serviços e aumentar a segurança do paciente nos respectivos serviços de saúde. Com incentivo internacional, o tema da 
segurança do paciente pautou ações da vigilância sanitária, em nível federal, com a elaboração do PNSP13; a aprovação da RDC Anvisa $n^{\circ} 36 / 2013^{15}$ - que dispõe sobre ações de segurança do paciente nos serviços de saúde; e a elaboração de recomendações de segurança e qualidade para os Serviços de Atenção Materna e Neonatal'7.

No modelo teórico-lógico das ações de vigilância sanitária, o componente "monitoramento do risco sanitário" abrange o subcomponente segurança do paciente ${ }^{5}$. A partir deste encadeamento, depreende-se que a segurança materna e a segurança neonatal ramificam-se da segurança do paciente, como especialidades inerentes aos serviços que tenham como público-alvo mulheres em estado gravídico e puerperal, bem como, seus filhos recém-nascidos, mas com ações coordenadas pela Visa.

A atenção materna e neonatal constitui área prioritária no que tange à segurança do paciente ${ }^{7}$. Isso porque ocorrem cerca de três milhões de nascimentos por ano no Brasil ${ }^{16}$, sendo que $98,5 \%$ são realizados em hospitais ou maternidades ${ }^{17}$. Portanto, a população exposta a procedimentos relativos aos serviços de atenção materna e neonatal corresponde a aproximadamente seis milhões de mulheres e crianças. Dados reportados no documento analisado ainda referem que os procedimentos obstétricos correspondiam à terceira causa de internamentos hospitalares no Sistema único de Saúde (SUS) em 2012; ano em que se computaram 218.418 internações devido a problemas oriundos do período perinatal ${ }^{7}$.

Além disso, argumenta-se que o modelo de atenção obstétrica predominante no Brasil preconiza a realização rotineira de intervenções (como episiotomia, cesárea e uso de ocitocina) no processo de parturição que expõem mulheres e crianças a riscos desnecessários, podendo culminar em mortes maternas e neonatais que poderiam ser evitadas por adequada assistência ${ }^{7,18}$. A qualidade da atenção torna-se um dos principais determinantes dos desfechos de saúde materna e perinatal, tendo em vista que quase todas as mulheres têm acesso aos serviços de assistência à gestação e ao parto no Brasil ${ }^{19}$.

O documento da Anvisa estudado oferece orientações para construção e reforma de unidades de atenção materna e neonatal, organização e estruturação desses serviços e elaboração de sistemas de segurança do paciente. 0 objetivo das orientações é "promover uma atenção obstétrica e neonatal de qualidade que reduza os agravos resultantes do próprio processo reprodutivo e minimize os danos relacionados ao processo assistencial"7. 0 alcance deste objetivo pressupõe a superação da dicotomia segurança versus humanização. Pois, a concepção transversal a todas as recomendações prescritas no documento é de que a segurança materna e neonatal deve incluir aspectos de ordem emocional, social e cultural. Com esse pressuposto, a definição de dano decorrente do processo assistencial é ampliada, conforme se lê abaixo:

$\mathrm{Na}$ atenção materna e neonatal deve prevalecer uma concepção multidimensional onde, tanto os eventos adversos que comprometem a estrutura ou função do corpo, como lesões, incapacidade ou disfunção ou mesmo a morte, assim como o sofrimento social, psicológico, moral e cultural devem ser enquadrados na categoria de danos ao paciente ${ }^{7}$.

Com foco na segurança e na humanização, a meta da Anvisa é "[...] contribuir com os esforços governamentais de redução da mortalidade e morbidade materna e neonatal no país, além da redução dos danos físicos ou psicológicos resultantes dos erros e práticas inadequadas dos serviços assistenciais". O PPQSAMN desdobra-se em dois componentes: segurança materna e segurança neonatal ${ }^{7}$.

O conteúdo do PPQSAMN ancora-se na tríade: estrutura, processos e resultados, proposta por Avedis Donabedian para avaliação da qualidade da assistência à saúde. Com esses elementos demarcados pelo modelo lógico aqui proposto (Figura), foram identificados os elementos estruturais necessários ao funcionamento do PPQSAMN e agrupados em quatro espécies de recursos: 1) procedimentos e instruções aprovados e vigentes; 2 ) ambiente adequado aos serviços prestados conforme legislação pertinente ao nível de complexidade do serviço; 3) pessoal qualificado e treinado; 4) recursos financeiros, equipamentos, insumos, materiais e suporte logístico conforme legislação aplicável ao tipo de estabelecimento/serviço. Os elementos que compõem cada espécie de recurso estão descritos na Tabela.

Ao examinar a disponibilidade e a qualidade dos elementos estruturais, é possível detectar possíveis problemas e analisar de que forma essas dificuldades afetam a provisão do cuidado e os processos assistenciais ${ }^{7}$. A pesquisa qualitativa de Maia et al. ${ }^{20}$ realizou entrevistas com um responsável e um técnico de Visa de serviços de saúde e um coordenador da área de saúde da mulher de secretaria de saúde de três municípios, com localização no Norte, Nordeste e Sul do Brasil. A análise de conteúdo, relativa às respostas para a questão sobre características da qualidade dos serviços que atendem a saúde da mulher, utilizou as categorias de estrutura, processo e resultado. As entrevistadas enfatizaram aspectos relativos à estrutura, tanto em relação a insumos quanto aos recursos humanos necessários à assistência. Os achados de pesquisa são um indício de que há necessidade de capacitar os profissionais para reconhecer outros elementos que compõem a qualidade dos serviços de saúde, especialmente aqueles relativos às práticas e atividades que podem contribuir para a segurança materna e neonatal.

Especificamente quanto à assistência prestada no âmbito de unidades de terapia intensiva neonatais, estudo de Tomazoni et al. ${ }^{21}$ com 28 profissionais de enfermagem e medicina constatou que há um reconhecimento da importância da segurança do paciente e a compreensão de que as rotinas de trabalhos podem expor os recém-nascidos a riscos. Dentre os fatores que interferem na segurança do paciente, na perspectiva dos profissionais, estão: a infraestrutura inadequada do ambiente hospitalar, materiais e equipamentos antigos e sem manutenção, e dificuldade de substituição de profissionais que faltam ao serviço - o que acarreta aumento da jornada de trabalho e fadiga. Os entrevistados também apontaram que não há 
PROGRAMA PARA A PROMOÇÃO DA QUALIDADE E SEGURANÇA DA ATENÇÃO MATERNA E NEONATAL (PPQSAMN)

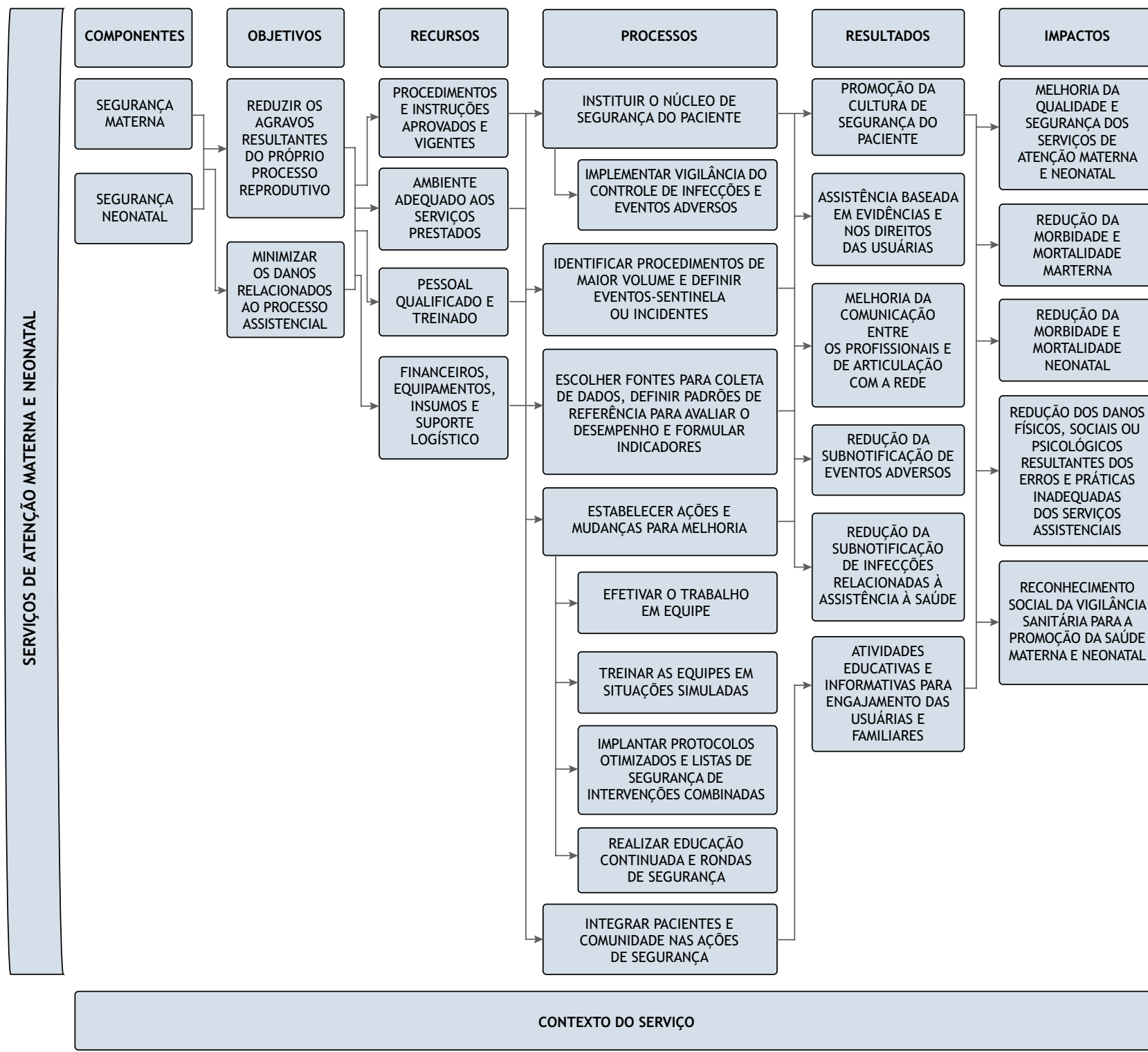

Fonte: Anvisa?

Figura. Modelo lógico do Programa para Promoção da Qualidade e Segurança na Atenção Materna e Neonatal.

incentivo para a comunicação das falhas ocorridas por parte da gestão e que, na hipótese de erro, não são propiciados espaços para discussão e aprendizado coletivo.

Os processos, por sua vez, reúnem o conjunto de atividades desempenhadas pelos diversos profissionais envolvidos direta ou indiretamente com a assistência obstétrica e neonatal. As recomendações para a implementação do PPQSAMN contemplam ações retrospectivas, no sentido de examinar práticas já realizadas para diagnosticar falhas, e ações prospectivas, para melhorar a performance da assistência conforme padrões pautados em evidências científicas e nos direitos humanos das/os usuárias/os. Além disso, estratégias podem ser pensadas para incorporar inovações tecnológicas e pesquisas.
Salienta-se, ainda, que o "foco das atividades é no sistema e trabalho em equipe e não nos indivíduos. Sanções e punições não são adequadas, além de não serem efetivas"7. Ou seja, estimula-se que os processos assistenciais sejam verificados com o intuito de gerar aprendizados que valham para o serviço como um todo. A segurança, como atributo da qualidade, é propiciada tanto pelas ações de prevenção dos eventos adversos e danos causados pelo processo assistencial, como pelas ações positivas que buscam implantar melhorias e mudanças que reforcem a confiabilidade de trabalhadores e usuárias/os nos serviços de atenção materna e neonatal.

É com esse sentido que deve ser criado o Núcleo de Segurança do Paciente (NSP), voltado para a atenção materna e neonatal ${ }^{13,15}$. 
Tabela. Recursos necessários ao funcionamento do Programa para Promoção da Qualidade e Segurança na Atenção Materna e Neonatal.

\begin{tabular}{|c|c|}
\hline \multirow{9}{*}{$\begin{array}{l}\text { Procedimentos e instruções } \\
\text { aprovados e vigentes }\end{array}$} & $\begin{array}{l}\text { - licença sanitária atualizada, expedida pela Visa local e dados atualizados no Cadastro Nacional dos Estabeleci- } \\
\text { mentos de Saúde }\end{array}$ \\
\hline & - regimento interno ou equivalente atualizado \\
\hline & - atividades/serviços terceirizados formalizados por contrato com prestadores portadores de licença sanitária \\
\hline & $\begin{array}{l}\text { - normas, protocolos e rotinas técnicas escritas e atualizadas com base em evidências científicas, de fácil acesso } \\
\text { a toda a equipe de saúde }\end{array}$ \\
\hline & $\begin{array}{l}\text { - comissões e comitês em funcionamento conforme legislação (por exemplo: comitê de mortalidade materna, } \\
\text { fetal e neonatal) }\end{array}$ \\
\hline & - documento formal sobre serviços de referência e contrarreferência \\
\hline & - mecanismos para identificação e controle de acesso dos trabalhadores, pacientes, acompanhantes e visitantes \\
\hline & • organização, manutenção e disponibilidade da documentação do estabelecimento \\
\hline & • organização das informações de atenção às usuárias (prontuários etc.) \\
\hline \multirow{8}{*}{$\begin{array}{l}\text { Ambiente adequado aos serviços } \\
\text { prestados conforme legislação }\end{array}$} & - projeto básico de arquitetura aprovado pela Visa \\
\hline & - instalações prediais conforme notas técnicas \\
\hline & - acessibilidade \\
\hline & - qualidade/continuidade do abastecimento de água \\
\hline & - garantia de continuidade de energia elétrica \\
\hline & - controle de pragas e vetores \\
\hline & - manejo dos rejeitos de acordo com plano de gerenciamento de resíduos de serviços de saúde \\
\hline & - limpeza dos espaços interiores/exteriores \\
\hline \multirow{7}{*}{ Pessoal qualificado e treinado } & - responsável técnico e substituto habilitados pelos respectivos conselhos de classe \\
\hline & - equipe dimensionada conforme normas/leis vigentes \\
\hline & - profissionais legalmente habilitados \\
\hline & - educação permanente \\
\hline & - avaliação e registro periódico da saúde ocupacional dos trabalhadores \\
\hline & - orientação para prevenção dos riscos de acidentes de trabalho \\
\hline & $\begin{array}{l}\text { - treinamento da equipe para assistência humanizada e segura, atuação com base em protocolos institucionais, } \\
\text { identificação e manejo das complicações obstétricas, atendimento às urgências e emergências }\end{array}$ \\
\hline \multirow{4}{*}{$\begin{array}{l}\text { Financeiros, equipamentos, } \\
\text { insumos, materiais e suporte } \\
\text { logístico conforme legislação }\end{array}$} & - equipamentos de proteção individual \\
\hline & - sistemas de informação \\
\hline & - insumos de acordo com o nível de complexidade e demandas do serviço \\
\hline & - garantia, em tempo integral, de acesso aos recursos assistenciais, diagnósticos e terapêuticos de apoio \\
\hline
\end{tabular}

Fonte: Anvisa ${ }^{7}$.

Sua composição deve ser multiprofissional e reunir as condições necessárias para coordenar ações retrospectivas e prospectivas que culminem na promoção de uma cultura de segurança nos serviços. É de competência do NSP a notificação dos eventos adversos (por exemplo: reação adversa a medicamento, incidente relacionado ao processo assistencial) e queixas técnicas de produtos sujeitos à vigilância sanitária (por exemplo: vacinas, artigos e equipamentos médico-hospitalares). A notificação à Visa não exime a responsabilidade pela investigação epidemiológica e tomada de medidas para o controle da situação.

Dentre as estratégias elencadas, sugere-se primeiro a identificação de prioridades de melhoria, com base na verificação dos procedimentos de maior volume, como o número médio de partos normal e cesárea realizado pelo estabelecimento. De acordo com Anvisa ${ }^{7}$, é importante definir os eventos-sentinela ou incidentes, cujas causas precisam ser investigadas e analisadas e demandam notificação. Dentre os eventos-sentinela maternos sugeridos constam: parto eletivo programado; readmissão materna não planejada dentro de 14 dias pós-parto; remoção, lesão ou reparo não planejado de órgão; complicações anestésicas; morte materna etc. Os eventos-sentinela fetais/neonatais podem contemplar: lesão fetal em cesariana, anomalia fetal não diagnosticada, mortes fetais e neonatais com peso menor ou igual a 500 gramas, entre outras. Orienta-se, também, a definição de eventos-sentinela relativos à organização do serviço que podem comprometer a qualidade da assistência, por exemplo: prontuário não disponível, reclamação de usuária/o, infecção relacionada à assistência. Os eventos-sentinela devem ser congruentes ao nível de complexidade e demanda dos serviços.

Outras atividades recomendadas pela Anvisa ${ }^{7}$ envolvem a verificação das fontes disponíveis para coleta de dados (sistemas de informação, prontuários, manifestações em ouvidoria etc.), estabelecimento de padrões de referência para a assistência e decisão quanto aos indicadores que possibilitem avaliar o desempenho do serviço. Os indicadores podem contemplar os recursos, os processos e os resultados almejados, desde que haja coerência com os padrões de referência estabelecidos. Exemplos de indicadores pertinentes: "taxa de óbitos decorrentes de falha durante a assistência", "percentual de incidentes por grau do dano", "incidência de infecção de sítio cirúrgico de parto por cesárea”. 
0 estudo de Moraes et al. ${ }^{22}$, realizado em um hospital-escola do interior do estado de São Paulo, verificou junto aos dados do NSP que, dentre as 89 notificações de eventos adversos, 30,3\% estavam relacionadas ao trauma mamilar, $25,8 \%$ a falhas na comunicação, 20,2\% à administração de medicamentos e 14,6\% à identificação. 0 estudo também concluiu que a falta de indicadores para avaliar o desempenho da atenção materna e neonatal dificulta a implementação de uma cultura de segurança na instituição.

Na hipótese de falhas ou de assistência prestada abaixo dos parâmetros predefinidos, recomenda-se investigar e monitorar as causas que contribuíram para o desfecho negativo. A maior parte dos eventos adversos ou incidentes possui causas multifatoriais, reconhecê-las a partir do ponto de vista institucional é mais efetivo que buscar culpados individualmente. Seguindo o modelo teórico-lógico da Anvisa ${ }^{5}$, esse processo envolve o "monitoramento dos níveis de infecção relacionados à assistência à saúde" e o "monitoramento dos eventos adversos da assistência à saúde". São várias as metodologias que podem ser úteis aos processos de investigação e monitoramento, e o documento da Anvisa ${ }^{7}$ oferece como sugestão os sete passos para a Análise da Causa-Raiz.

Ato contínuo ao monitoramento, deve-se estabelecer ações e mudanças para a melhoria. Essas ações decorrem de uma série de atividades concomitantes dependentes do efetivo trabalho em equipe; treinamentos em situações simuladas; elaboração de diretrizes clínicas baseadas em evidências; implantação de protocolos otimizados, listas de seguranças e intervenções combinadas; e processos de educação continuada e de rondas de segurança ${ }^{7}$.

0 trabalho multiprofissional é essencial, mas ainda incompleto sem a integração e engajamento dos pacientes, familiares e da comunidade para a efetivação de uma cultura da segurança. Ainda que não liste efetivamente essa ação como parte das estratégias para garantia do PPQSAMN, depreende-se da leitura de todo o documento ${ }^{7}$, a necessidade de integrar pacientes, familiares e a comunidade nas ações de segurança. Isso pode ocorrer por meio de atividades educativas sobre uso seguro de medicamentos, higienização das mãos, tosse com etiqueta, prevenção e controle de infecções. Ademais, para além do que está no documento, é profícuo estabelecer uma cultura de identificação e notificação por parte dos pacientes, com serviços de ouvidorias ativas e possibilidades efetivas de inclusão da comunidade nos processos de gestão de segurança ${ }^{23,24}$.

Outras ações podem ser executadas, dependendo do tipo e da demanda assistencial do serviço, considerando também o manual Assistência Segura: uma reflexão teórica aplicada à pratica ${ }^{25}$.

A partir desses processos, os resultados englobam os efeitos de curto e médio prazo. Compreende-se como resultados a redução de subnotificação de infecções relacionadas à assistência à saúde e a redução das subnotificações de eventos adversos, o que contribuirá para a "redução do número de serviços de saúde classificados como médio-alto e de alto risco" em nível nacional ${ }^{5}$. Outros resultados relacionados ao conjunto dos processos que compõem o programa são: a melhoria da comunicação entre os profissionais do serviço, da articulação da rede assistencial, e do engajamento de pacientes, familiares e a comunidade ${ }^{5,7,13}$.

A implementação do PPQSAMN nos serviços de atenção materna e neonatal objetiva a promoção da cultura de segurança do paciente e da humanização da atenção obstétrica e neonatal que pressupõe a assistência baseada em evidências com o respeito aos direitos humanos das mulheres, recém-nascidos e de suas famílias. Conforme o conceito formulado por Pittrof et al. ${ }^{26}$, a qualidade nos serviços de atenção materna envolve a conjugação de "cuidados mínimos" para maior parte das mulheres e recém-nascidos e de alta complexidade para aqueles que precisarem. Haja vista que a maior parte das gestações, partos e nascimentos transcorrem sem a necessidade de intervenções, os “cuidados mínimos" correspondem às boas práticas de assistência obstétrica e neonatal, que preconizam postura não invasiva e a realização de intervenções quando houver indicação baseada em evidências. Isso porque a prática rotineira de procedimentos invasivos expõe a mulher e o bebê a maiores riscos de eventos adversos relacionados à assistência. Qualquer que seja o nível de complexidade da atenção, devem ser considerados aspectos sociais, culturais e emocionais relativos ao nascimento.

Também se pontua como resultado esperado a melhoria da comunicação entre os profissionais. Isso porque a efetividade das ações elencadas preconiza o trabalho em equipe. A cooperação entre profissionais de posições hierárquicas e funções distintas é um desafio permanente para os serviços de saúde. 0 fortalecimento do trabalho em equipe constitui estratégia essencial para o fomento de uma cultura de segurança, haja vista que ações descoordenadas e dificuldades de comunicação entre os profissionais estão no cerne de muitos eventos adversos ou incidentes no âmbito da atenção materna e neonatal ${ }^{7}$.

Da articulação entre recursos adequados, processos coerentes com a segurança do paciente e a humanização e resultados previstos em âmbito institucional, é possível antever impactos para a população de um modo geral. São efeitos que demonstram como cada instituição pode contribuir, em sentido amplo, para: a) melhoria da qualidade e segurança dos serviços de atenção materna e neonatal; b) redução da morbidade e mortalidade materna e neonatal; c) redução dos danos físicos, sociais ou psicológicos resultantes dos erros e práticas inadequadas dos serviços assistenciais; e d) reconhecimento social da vigilância sanitária para a promoção da saúde materna e neonatal. Toda a constituição do modelo lógico reforça a necessidade de integração entre os órgãos de Visa e os serviços de atenção materna e neonatal.

\section{CONCLUSÕES}

A constituição do modelo lógico apresentado procurou integrar uma das atribuições da vigilância sanitária para promoção da saúde materna e neonatal, especificamente a segurança do paciente prevista no modelo teórico-lógico da Anvisa ${ }^{5}$. A análise teve como foco o documento Serviços de Atenção Materna e Neonatal: segurança e qualidade, que propõe um PPQSAMN. 
Do mesmo modo que o PPQSAMN pode ser executado pelos serviços de assistência obstétrica e neonatal, mediante adaptações conforme o nível de complexidade e demandas do serviço, espera-se que o modelo lógico ora proposto possa contribuir para atividades de avaliação das equipes gestoras dos serviços pertinentes.

0 modelo foi construído com base na tríade estrutura, processos e resultados, de acordo com Donabedian, e buscou formas de sintetizar e organizar o conjunto de recomendações para segurança materna e neonatal. Teve a preocupação de perceber a importância do trabalho multiprofissional e a integração de pacientes, familiares e comunidade para a promoção de cultura de segurança do paciente.

Ainda que não explorado profundamente no documento base ${ }^{7}$, acredita-se que o fortalecimento do controle social pode potencializar os processos de avaliação dos serviços, bem como o reconhecimento e valorização social da Visa para a promoção da saúde materna e neonatal. A experiência das "ouvidorias ativas" pode contribuir com os mecanismos de monitoramento, por exemplo, dos incidentes ou eventos adversos relacionados à assistência à saúde.

Por fim, destaca-se a necessidade de articulação e de complementaridade dos serviços de Visa e de assistência obstétrica e neonatal. Salienta-se que as recomendações da Visa analisadas estão alinhadas com a Política Nacional de Atenção Integral a Saúde da Mulher e a Política Nacional de Atenção Integral à Saúde da Criança. Principalmente com o Programa de Humanização no Pré-Natal e Nascimento que propõe práticas obstétricas e neonatais baseadas nas melhores evidências científicas e com a centralidade da mulher e sua família, com efetivação do respeito aos direitos humanos de usuárias/os.

\section{REFERÊNCIAS}

1. Brasil. Lei $\mathrm{N}^{\circ} 9.782$, de 26 de janeiro de 1999 . Define 0 sistema nacional de vigilância sanitária, cria a agência nacional de vigilância sanitária, e dá outras providências. Diário Oficial União. 27 jan.1999.

2. Maia C, Guilhem D, Lucchese G. Integração entre vigilância sanitária e assistência à saúde da mulher: um estudo sobre a integralidade no SUS. Cad Saude Publica. 2010;26(4):682-92. https://doi.org/10.1590/S0102-311X2010000400011

3. Fernandes RZS, Vilela MFG. Estratégias de integração das práticas assistenciais de saúde e de vigilância sanitária no contexto de implementação da rede cegonha. Cienc Saude Coletiva. 2014;19(11):4457-66. https://doi.org/10.1590/1413-812320141911.21662013

4. Tomazoni A, Rocha PK, Kusahara DM, Souza AIJ, Macedo TR. Avaliação da cultura de segurança do paciente em terapia intensiva neonatal. Texto Contexto Enferm. 2015;24(1):1619. https://doi.org/10.1590/0104-07072015000490014

5. Agência Nacional de Vigilância Sanitária - Anvisa. Avaliação das ações de vigilância sanitária: uma proposta teóricometodológica. Brasília: Agência Nacional de Vigilância Sanitária; 2017.

6. Felisberto E, Brito RL, Barca DAAV, Martins MAF, Oliveira AKNBM, Souza NC et al. Modelagem avaliativa para a construção de indicadores de efetividade das ações de vigilância sanitária no Brasil. Rev Bras Saude Mater Infant. 2018;18(3):665-76. https://doi.org/10.1590/1806-93042018000300011

7. Agência Nacional de Vigilância Sanitária - Anvisa. Serviços de atenção materna e neonatal: segurança e qualidade. Brasília: Agência Nacional de Vigilância Sanitária; 2014.

8. Bezerra LCA, Cazarin G, Alves CK. Modelagem de programas: da teoria à operacionalização. In: Samico I, Felisberto E, Figueiró AC, Frias PG, organizadores. Avaliação em saúde: bases conceituais e operacionais. Rio de Janeiro: Medbook; 2010. p. 65-78.
9. McLaren J. Evaluating programs for women: a genderspecific framework. Winnipeg: Prairie Women's Health Centre of Excellence; 2000.

10. Medina MG, Silva GAP, Aquino R, Hartz ZMA. Uso de modelos teóricos na avaliação em saúde: aspectos conceituais e operacionais. In: Hartz ZMA, Silva LMV, organizadores. Avaliação em saúde: dos modelos teóricos à prática na avaliação de programas e sistemas de saúde. Rio de Janeiro: Fundação Oswaldo Cruz; 2005. p. 41-63.

11. Champagne F, Brousselle A, Hartz Z, Contandriopoulos AP. Modelizar as intervenções. In: Brousselle A, Champagne F, Contandriopoulos AP, Hartz Z, organizadores. Avaliação: conceitos e métodos. Rio de Janeiro: Fundação Oswaldo Cruz; 2011. p. 61-74.

12. Pinto ESG, Souza NL, organizadoras. Caminho para a avaliação em saúde: elaboração de modelos lógicos como passo inicial. Curitiba: CRV; 2017.

13. Ministério da Saúde (BR). Portaria $N^{\circ} 529$, de 1 de abril de 2013. Institui o programa nacional de segurança do paciente (PNSP). Diário Oficial União 2 abr 2013.

14. Agência Nacional de Vigilância Sanitária - Anvisa. Resolução RDC № 36, de 25 de julho de 2013. Institui ações para a segurança do paciente em serviços de saúde e dá outras providências. Diário Oficial União. 26 jul 2013.

15. Agência Nacional de Vigilância Sanitária - Anvisa. Resolução RDC № 36, de 3 de junho de 2008. Dispõe sobre regulamento técnico para funcionamento dos serviços de atenção obstétrica e neonatal. Diário Oficial União. 4 jun. 2008.

16. Instituto Brasileiro de Geografia e Estatística - IBGE. Estatísticas do registro civil. Rio de Janeiro: Instituto Brasileiro de Geografia e Estatística; 2018[acesso 3 jun 2020]. Disponível em: https://sidra.ibge.gov.br/ tabela/2679\#resultado 
17. Leal MC, Szwarcwald CL, Almeida PVB, Aquino EML, Barreto ML, Barros F et al. Saúde reprodutiva, materna, neonatal e infantil nos 30 anos do sistema único de saúde (SUS). Cienc Saude Coletiva. 2018;23(6):1915-28. https: //doi.org/10.1590/1413-81232018236.03942018

18. Bourguignon AM, Grisotti M. Concepções sobre humanização do parto e nascimento nas teses e dissertações brasileiras. Saude Soc. 2018;27(4):1230-45. https://doi.org/10.1590/S0104-12902018170489

19. Salgado HO, Souza JP, Sandall J, Diniz CSG. Patient safety in maternity care in Brazil: the maternity safety thermometer as a tool to improve the quality of care. Rev Bras Ginecol Obstet. 2017;39(5):199-201. https://doi.org/10.1055/s-0037-1602704

20. Maia CS, Freitas DRC, Guilhem D, Azevedo AF. Percepções sobre qualidade de serviços que atendem à saúde da mulher. Cienc Saude Coletiva. 2011;16(5):2567-74. https://doi.org/10.1590/S1413-81232011000500027

21. Tomazoni A, Rocha PK, Ribeiro MB, Serapião LS, Souza S, Manzo BF. Segurança do paciente na percepção da enfermagem e medicina em unidades de terapia intensiva neonatal. Rev Gaucha Enferm. 2017;38(1):1-13. https://doi.org/10.1590/1983-1447.2017.01.64996

22. Moraes AISM, Santos VL, Paes LBO, Parro MC. Qualidade e segurança na área da saúde materno-infantil: avaliação de eventos adversos. Cuidarte Enferm. 2019;13(1):32-7.

23. Almeida KJQ, Roure FN, Bittencourt RJ, Santos RMDB, Bittencourt FV, Gottems LBD et al. Ouvidoria ativa em saúde: avaliação da qualidade da atenção ao parto e nascimento. Rev Saude Publica. 2018;52(76):1-11. https://doi.org/10.11606/s1518-8787.2018052017291

24. Behrens R. Segurança do paciente e os direitos do usuário. Rev Bioet. 2019;27(2):253-6. https://doi.org/10.1590/1983-80422019272307

25. Agência Nacional de Vigilância Sanitária - Anvisa. Assistência segura: uma reflexão teórica aplicada à prática. Brasília: Agência Nacional de Vigilância Sanitária; 2013.

26. Pittrof R, Campbell OMR, Filippi VGA. What is quality in maternity care? An international perspective. Acta Obstet Gynecol Scand. 2002;81(4):277-83. https://doi.org/10.1034/j.1600-0412.2002.810401.x

\section{Agradecimentos}

O presente trabalho foi realizado com apoio da Coordenação de Aperfeiçoamento de Pessoal de Nível Superior-Brasil (CAPES) - Código de Financiamento 001.

Contribuição dos Autores

Bourguignon AM - Concepção, planejamento (desenho do estudo), aquisição, análise, interpretação dos dados e redação do trabalho. Hartz Z, Moreira D - Concepção, planejamento (desenho do estudo), aquisição, análise e interpretação dos dados. Todas as autoras aprovaram a versão final do trabalho.

Conflito de Interesse

Os autores informam não haver qualquer potencial conflito de interesse com pares e instituições, políticos ou financeiros deste estudo.

Esta publicação está sob a licença Creative Commons Atribuição 3.0 não Adaptada.

Para ver uma cópia desta licença, visite http://creativecommons.org/licenses/by/3.0/deed.pt_BR. 